confirmed its involvement in sex determination. Although these results show that $D M Y$ is necessary for male development, whether it is also sufficient remains to be seen.

Magdalena Skipper

\section{(0) References and links ORIGINAL RESEARCH PAPER Matsuda, M. et al. DMY is a Y-specific DM-domain gene required for male development in the medaka fish. Nature 417, 559-562 (2002) \\ FURTHER R Medaka - a model organism from the Fa East. Nature Rev. Genet. 3, 53-64 (2002)}

contain factors involved in X inactivation. However, chromosome-15 markers showed no association with the mutant phenotype in the 1.19 pedigree, indicating the presence of two distinct mutations. A lack of informative markers for the 1.19 pedigree prevented this mutation from being mapped further in this strain.

With this work, the authors show for the first time that autosomal factors are involved in the choice of which $\mathrm{X}$ chromosome to inactivate. But they still have some way to go before they can say what these factors are, and how they interact with each other and the X chromosome. Such future studies, however, are likely to be helped by new sequencing data from Chureau et al. on the mouse and bovine Xic regions. In a threeway comparison of this region between mouse, cow and human, these authors identified conserved coding and non-coding genes, conserved $\mathrm{CpG}$ islands and even conserved pseudogenes, in addition to four new genes. With Chureau et al.'s new insights into what the Xic consists of, Percec et al. should find themselves better placed to answer the questions that their results pose.

Jane Alfred

(1) References and links ORIGINAL RESEARCH PAPERS Percec, l. etal. Autosomal dominant mutations affecting $X$ inactivation choice in the mouse. Science $\mathbf{2 9 6}$ 1136-1139 (2002) | Chureau, C. et al. Comparative sequence analysis of the X-inactivation center region in mouse, human, and bovine. Genome Res. 12 894-908 (2002) FURTHER READING Avner, $P$ \& Heard, $E$. $X$-chromosome inactivation: counting, choice and initiation. Nature Rev. Genet. 2, 59-67 (2001) WEB SITES

Hunt Willard's lab:

http://genetics.gene.cwru.edu/willard Marisa Bartolomei's lab: http://wnw. med. upenn.edu/cellbio/faculty/bartolomei

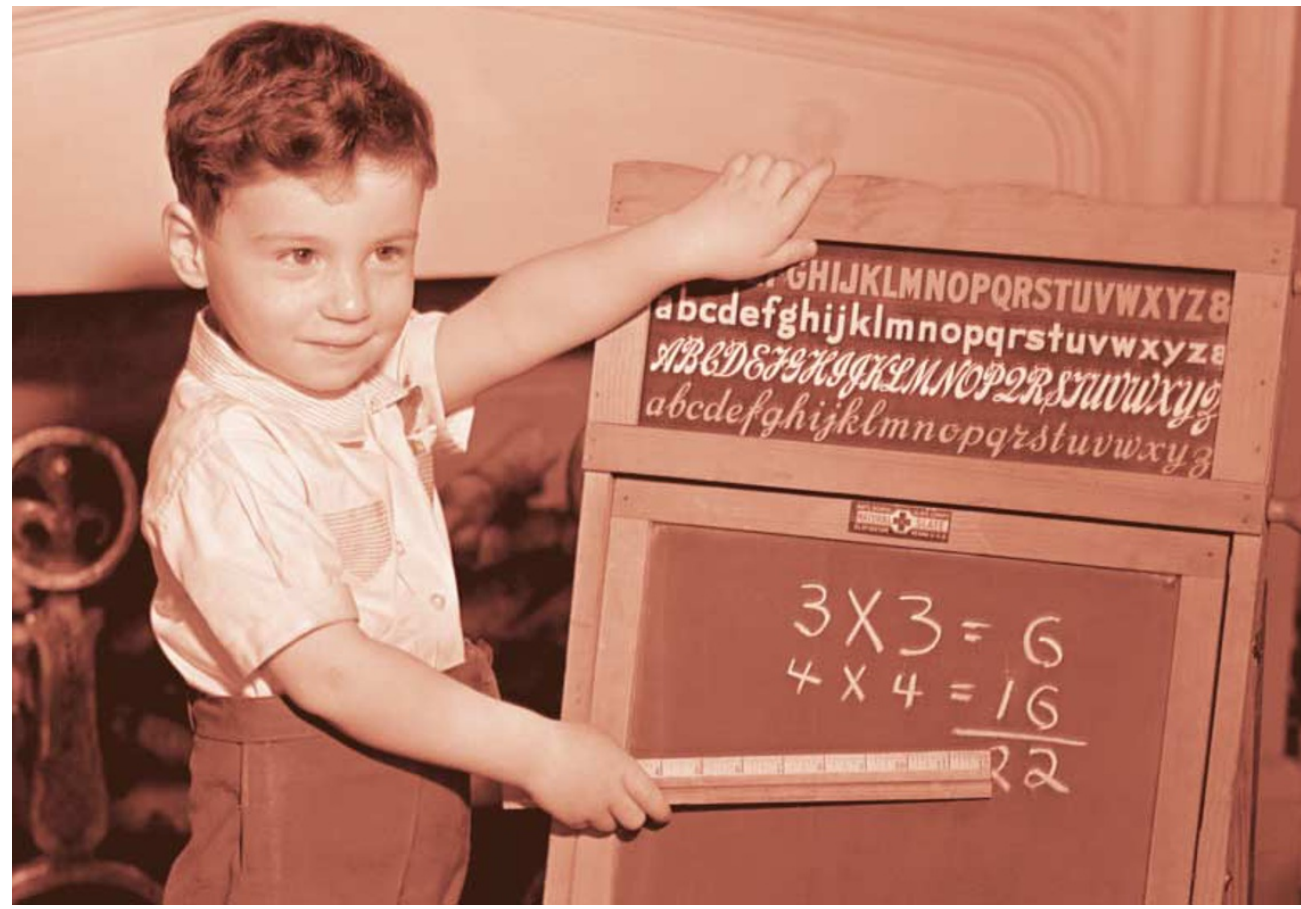

BEHAVIOURAL GENETICS

\title{
An old lesson in behaviour
}

Picking up someone else's project is rarely top of a researcher's wish list, especially if it has been around for more than four decades. But if the project happens to be that of Jerry Hirsch, then perhaps the idea is worth reconsidering. In the 1950s and 1960s, this 'drosophilist' sought to analyse the genetic basis of behaviour by artificially selecting lines of Drosophila that had an extreme preference for moving towards or against gravity in a vertical maze. Although he was able to establish, for the first time, that this so-called geotaxic behaviour - indeed any behaviour - has a genetic basis, getting to the underlying genes just wasn't possible at that time. However, by applying cDNA microarray experiments and mutant analysis to the original lines generated by Hirsch, Daniel Toma, along with Ralph Greenspan, Kevin White and Jerry Hirsch himself, have now partly realized the original researcher's aim by identifying three genes involved in fly geotaxic behaviour. The genetic basis of any selected phenotype is rather impenetrable, even today, making this work all the more remarkable.

If Hi (flies that like to go 'up') and Lo (flies that like to go 'down') lines behave differently, then the genes involved in this divergent phenotype are probably differently expressed in the two lines. In a microarray experiment to assess this, the authors identified 250 genes whose expression levels differed at least twofold between the two lines.

Toma et al. decided to pursue only those candidates from their microarray analysis for which mutants with neurological defects already exist. This left them with four mutant lines cryptochrome (cry), Pendulin (Pen), Pigment- dispersing factor (Pdf ) and prospero (pros) which were tested for their preference to go up or down. The geotaxic score of three of the mutants, the exception being pros, was significantly different from that of wild-type flies and correlated with the difference in mRNA levels seen in the selected $\mathrm{Hi}$ and Lo lines. The dosage effect of gene expression on behaviour was also tested by generating transgenic flies that expressed wild-type $P d f$ and pros in backgrounds with varying copies of the endogenous transcript. Although altering the level of pros had no significant effect on the geotaxic score (as predicted from the mutant data), altering the dosage of $P d f$ produced a graded effect, which differed between the sexes.

This file might have been an old one but, despite the qualitative advance reported here, it still isn't closed. How do Pen, Pdf and cry influence behaviour, as their functions give us little clue? How do we identify the remaining genes, which are probably pleiotropic and of small effect? Regardless of the outstanding questions, this work shows that behaviour can be genetically dissected by combining classical quantitative analysis, genomic approaches and mutant characterization - a new 'modern synthesis' for understanding the genetic architecture of complex traits.

Tanita Casci

\section{(2) References and links}

ORIGINAL RESEARCH PAPER Toma, D. P. et al. Identification of genes involved in Drosophila melanogaster geotaxis, a complex behavioral trait. Nature Genet. 3 June 2002 (10.1038/ng893) WEB SITES

Kevin White's lab:

http://www.med.yale.edu/bbs/gendev/faculty/white.htm Jerry Hirsch's lab:

http://www.psych.uiuc.edu/people/faculty/hirsch.htm

Ralph Greenspan's lab: www.nsi.edu 